\title{
The impact of e-commerce development on electronic centralized procurement model
}

\author{
Chen Zhang \\ Engineering Management, ZhengZhou University, Kexue Avenue, Zhengzhou, China
}

Keywords: State-owned enterprises, Centralized procurement, Electronic platform, Future development, Risk management

\begin{abstract}
The SOE reforms and the "supply-side reform" are the key words of the past few years. In 2015, the State-owned Assets Supervision and Administration Commission requested state-ownedenterprises to comprehensively build an electronic centralized procurement platform, and electronic centralized procurement became the mainstream mode of state-owned enterprise procurement. This paper combines the development trend of e-commerce, explores a mainstream management model, and discusses the future development of this model from the aspects of controlling risks and improving efficiency.
\end{abstract}

\section{Introduction}

\subsection{Overview of E-Commerce Development}

As China's economic level continues to increase and consumers' acceptance of online shopping has increased, the market size of the e-commerce industry has continued to expand. The ecommerce industry has created a third-party e-commerce platform that can aggregate the huge resources of buyers and sellers through the means of the Internet, breaking the time and space restrictions of traditional social retail transactions and improving the transaction efficiency of the overall social retail (Yao Li, Mingyan $\mathrm{Li}$, 2006).The construction of the e-commerce business ecosystem helps the brand to expand sales channels and reduce operating costs. On the other hand, it helps consumers expand their purchasing channels, improve information transparency, and effectively improve social and economic benefits and social values.

At present, the deep integration of e-commerce and industrial development has been continuously increased, accelerating the formation of a new trend of economic competition. In thecase that comprehensive e-commerce websites have occupied most of the market in the comprehensive B2B field, more and more industry e-commerce websites have sought the development of specialized sub-sectors outside the integrated website market. E-commerce is indepth integration with traditional industries, and the two promote each other and coordinate development.

The continuous popularization of e-commerce will directly lead to the development of industries such as animal flow, finance and IT, and drive the development of sub-formats in the e-commerce ecosystem, such as third-party payment, electronic certification, network information security, and network insurance. A large number of e-commerce service providers, with the development of 
various nodes in the animal flow supply chain. Auxiliary e-commerce services have spawned some new service industries. The development of e-commerce and international e-commerce in enterprise supply chain will drive the development of e-commerce service industry. The development of the ecommerce service industry will also occupy more market share in the next few years.

\subsection{Overview of Electronic Centralized Purchasing Management}

E-procurement is a business processing method that replaces traditional paperwork with a computer system and completes procurement through a network support system, also known as online procurement(Wei Xiong, Ming Xu, 2006).Its basic feature is to establish e-commerce trading platform, publish procurement information, or actively find suppliers on the Internet, find products, online ordering, price comparison, online bidding to achieve online ordering, online payment, and finally through the logistics system for goods distribution, complete the entire transaction process, belongs to the B2B mode of e-commerce.

The centralized procurement of electronic products is a combination of centralized procurement and electronic procurement, and procurement is carried out by means of electronic procurement and centralized procurement (Qiaoyan Cao,2007). The requirements for new economic work in the centralized procurement of electronic products first appeared in the procurement work of government agencies, usually by the procurement agency to implement specific bidding work. The centralized procurement management of state-owned enterprises is an important part of the reform and restructuring of state-owned enterprises led by the SASAC, and is quite different from the centralized procurement management of the government. Its predecessor was a centralized procurement platform. After the 18th National Congress of the Communist Party of China, the State-owned Assets Supervision and Administration Commission issued the "Basic System for Regulating the Internal Control of State-Owned Enterprises." Central enterprises began to try to implement the centralized procurement management system and achieved initial success. In 2015, the State-owned Assets Supervision and Administration Commission issued the "Notice on Carrying out Procurement Management to Enhance Matters Related to Benchmarking Work”, requiring central enterprises to comprehensively build a unified electronic centralized procurement platform and integrate the original procurement activities into a unified management system. In the same year, a number of central enterprises completed the initial construction of the electronic centralized procurement platform, and gradually improved in the follow-up work. Up to now, a number of central enterprises have formed a variety of unique electronic centralized procurement management systems, and promoted to other state-owned enterprises.

\section{The significance of centralized electronic procurement for project management}

Electronic centralized procurement management has two advantages. First, the advantages of openness, informatization and transparency of electronic platforms: e-procurement expands the range of suppliers' choices, and enterprises can choose the best suppliers of quotations and services, breaking through the tradition. The limitation of the number of procurement suppliers to achieve the transformation of localized procurement to global procurement. At the same time, e-procurement automates the procurement process, enabling companies to find the right suppliers and items through the network, keep abreast of market conditions and inventory, prepare sales plans, purchase items online, and purchase orders and purchased items. In-transit, ledger and inventory management, automatic statistical analysis of procurement, improve the ability of enterprises to respond to market demand. The second is the advantages of standardization, integration and institutionalization of centralized procurement. 


\subsection{Standardize Purchasing Behavior}

The most direct advantage of electronic centralized procurement management is to regulate daily purchasing behavior. The implementation of centralized procurement can reduce the frequency of procurement, make a large number of procurement activities happen in a specific department or company, save a lot of supervision links, and make the supervision behaviors targeted; the application of electronic platform makes any procurement behavior transparent It is well documented, and the situation of information inequality is greatly reduced. At the same time, avoid the black-box operation behavior. The black-box operation behavior in decentralized procurement has always plagued the company's standardized operation. The centralized procurement of materials makes the procurement environment transparent, makes the procurement behavior open, fair and justice, and promotes the democratic decision-making, open operation, process control, and the whole process of the business operation, which is conducive to the enterprise to carry out anticorruption construction.

\subsection{Improve Project Quality}

The enterprise publishes the information of the purchased products on the Internet, and the supplier logs in the catalogue information of the qualifications, products, services, etc. in the enterprise system for the enterprise to evaluate. By establishing a system and maintenance system for the enterprise, for the enterprise, he can more closely control the entire procurement process, limit the types and specifications of the products required in the catalog, and set different procurement authority. It is easy to find the most suitable supplier by systematic evaluation of multiple suppliers in the system. Concentrated procurement behavior is accompanied by a centralized review, and the quality and acceptance of the subject matter of the procurement will receive more supervision. When the scattered procurement is avoided, the narrow scope of the audit corresponding to the single purchase behavior makes the procurement of the wrong subject matter, the entry object is unqualified, and the procurement process audit has been reduced. The source material, engineering, and service are controlled from the source. The quality of the service indirectly improves the quality of the project.

\subsection{Reduce Procurement Costs}

Zeroing into a centralized purchasing model makes the amount of each purchase behavior much higher than the original. With normal market rules, a large number of centralized procurement can enable suppliers to make more profits and obtain lower purchase prices. Cost reduction and synergy are huge. In the modern market environment, scale advantage often means price advantage. The implementation of centralized procurement can aggregate scattered procurement requirements into huge purchases, which is convenient for companies to increase their chips and negotiate purchase costs (Lusheng Lin, 2009). At the same time, centralized procurement is more convenient for enterprises to effectively control procurement quality and logistics distribution: concentrating procurement to suppliers with strong brands and strong strength, can effectively avoid the quality risks of purchasing materials; the use of e-commerce platform makes procurement information more transparent More potential suppliers can participate in the procurement process itself, making business competition more intense and further reducing costs.

\subsection{Promote Enterprise Transformation And Upgrading}

The implementation of centralized procurement means the establishment of a brand-new 
management system. It is not a simple internal system reform, but the optimization and reconstruction of the supply chain system in the economic operation of the enterprise, and at the same time it drives the information construction, execution ability, capital management ability, etc. Comprehensive progress in all aspects. In terms of information, centralized procurement requires an efficient information feedback system to cope with complex procurement behaviors, distribution behaviors and logistics behaviors. In terms of management and control capabilities, centralized procurement can strengthen the headquarters of the sub-branch in terms of strategic guidance and operational processes. Guidance and monitoring; in terms of fund management, it will further improve financial capabilities such as corporate budget management and capital cost control.

The current competition between enterprises and enterprises has gradually shifted from competition in the market and brands to competition in the supply chain. As a new management idea, supply chain management is one of the most important developments in the field of enterprise management in recent years. Supply chain management is to use the systemized and centralized thinking to coordinate the entire supply chain, and integrate the logistics, information flow and capital flow in the supply chain to maximize the overall benefit of the supply chain. Centralized procurement is to implement this systemized and integrated thinking to integrate internal and external resources of the enterprise. It can not only effectively allocate resources, but also realize the whole process management of the supply chain. The implementation of centralized procurement is conducive to the adjustment of business models in the direction of supply chain management, to adapt to changes in the competitive environment of enterprises (Jinghua Xiao, KangXie, YaoWu, Xuehua Liao, 2015).

\subsection{Risk Management}

\subsubsection{Capital Risk}

Centralized procurement has higher planning requirements for project needs, effectively reducing sudden purchases, making the use of funds more accurate, reducing the use of large amounts of funds, and effectively avoiding cash flow risks in capital risks.

\subsubsection{Human Resource Risk}

The establishment of a unified rear centralized management platform can greatly reduce the frontline human resource requirements, improve the project's ability to resist personnel movement, and reduce labor costs; at the same time avoid excessive personnel participation in core business information and reduce human resource risks.

\subsubsection{Institutional Risk}

Each project has its own particularity. It needs to establish a management system according to its own needs. If there are too many independent systems, it will generate institutional risks. Centralized management can well avoid this problem.

\subsubsection{Market Risk}

Each material in each region has its own unique market characteristics. The centralized procurement platform can simultaneously face multiple market cultures, learn more experiences and resist different market culture shocks. At the same time, centralized procurement will bring uniform supply. Business management reduces the investigation of unfamiliar suppliers, reduces the possibility of inferior suppliers, and enhances the ability to withstand different market environments 
and risks.

\section{The problem of centralized electronic procurement management}

As a new type of procurement management model, electronic centralized procurement management has formed a variety of different management systems after several years of rapid development. However, there are still some shortcomings, especially some common problems, which are manifested in:

\subsection{Poor Flexibility}

The electronic centralized procurement management process takes a long time, has a high demand for planning, and has insufficient ability to cope with unexpected situations. It has weak response to sudden new demand categories or sporadic consumable materials, and needs other measures to supplement and supplement. Improve the ability to respond to different situations. In the era of planned economy, the procurement behavior of enterprises is centralized procurement and planned procurement. After the market economy reform, procurement behavior becomes flexible and dispersed. Promoting centralized electronic procurement is not just about changing the procurement model back to the planned economy era, but while respecting the laws of the market.

\subsection{Market-Specific Behavior}

The overly public information makes the procurement behavior be targeted and destroyed. The open and sound procurement mechanism can not effectively clean up the malicious interference bidding behavior. Once the loopholes in the process are discovered, it will become a breakthrough for commercial competitors. Some enterprises lack centralized policies, regulations, supervision, incentives, etc. when they conduct centralized procurement and management of suppliers. The means are not strong enough, resulting in poor results.

\subsection{Imperfect Supporting System}

This kind of new management mode cannot be perfectly supported in the establishment of the company's superstructure system. The existing state-owned enterprises, especially the large enterprises directly under the central government, are partly derived from the institutions of the former ministries and commissions of the State Council. The large-scale enterprises affiliated to the various parts of the former State Council have strong government characteristics and strong planning characteristics. At the same time, after years of institutional and institutional changes, the original planned economic behavior began to transform into market economic behavior, and the procurement behavior changed from unified to decentralized, and procurement power was decentralized. Once the power is decentralized, it will be harder to recover it. In the process of promoting centralized procurement and tightening procurement authority, some people emphasize that the local interests of the production units are sacred and inviolable, and the flexibility of individual units must be guaranteed; the management model and system support are born at the same time, but the management model passes through the market. The test is step by step to maturity. In contrast, the renewal of the system is obviously backward, and the support for the management model is gradually weakened. Over time, it accumulates into a risk hazard. 


\section{Centralized procurement under the trend of e-commerce}

The development trend of e-commerce is mainly reflected in: the development environment of ecommerce will continue to improve, the driving force for development will continue to increase; the e-commerce application will reach new breadth and depth; the deep integration of e-commerce and industrial development will continue to increase, accelerating the formation of new economic competition. The situation; the e-commerce service industry is booming and has gradually become a new growth point for the national economy. How will these affect the emerging centralized electronics procurement?

\subsection{Development of Existing Advantages}

With the continuous deepening of the B2B model and the emergence of a more stable and convenient e-commerce portal or information portal, e-commerce will be more reliable and more suitable for the "standardization" requirements of enterprise electronic centralized procurement. On the one hand, institutionalize and streamline the procurement activities in the original electronic centralized procurement field, so that the supply and demand relationship is transparent and open, the market will survive the inferior, improve the quality of procurement materials and equipment, reduce procurement costs, and make procurement activities reasonable and compliant; In terms of broadening the scope of centralized electronic procurement, the traditional local equipment with strong locality, market opacity, professionalism or inconvenient centralized procurement will be included in the centralized procurement of electronic products, and the inherent supply chain will be broken through the updated supply model or the whole industry supply chain. The industrial barriers include the overall planning of materials and equipment in the project planning stage, enhancing the planning and cost control of the project.

With the development of the e-commerce service industry, not only the service demand can adopt the electronic centralized procurement mode, but also the supporting services driven by the industrial development will benefit the entire e-commerce field. For a wider range of procurement suppliers, it will inevitably need stronger support from the logistics system and payment system, drive the circulation of capital and materials and equipment, and establish a diversified supply and demand relationship, which not only promotes enterprise transformation and reform, but also enhances the company's ability to resist risks. Adding some flexibility and diversity to the original centralized electronic procurement model, enabling enterprises to better cope with the rapidly developing economic environment (Yue Zhang, Weidong Chen, Zhiping Fan, 2004). The improvement of the ability of state-owned enterprises to resist risks directly affects the stability of the entire social and economic situation and is related to people's livelihood.

\subsection{Complement of Shortcomings}

The deepening development of e-commerce has made the concept of e-commerce deeply rooted in people's minds. The rules and processes of the entire e-commerce system will be more standardized, universal and simplified, which will help lower the industry threshold, better drive market transformation, and transform enterprises. Relatively stable business model and environment, reducing business risk, market risk and human resource risk. Diversified supply and demand relationships and a more transparent procurement model provide enterprises with a flexible space to respond to changing markets and emergencies. At present, the intensity of local protectionism has gradually weakened. The rich supply model and strong logistics system have greatly increased the traditional supply radius of suppliers. The supply of large-scale construction equipment across multiple provinces has become the norm, maliciously disrupting the market to interfere with 
procurement specifications. Behavior will be gradually excluded from the excellent supply and demand relationship. The market model formed by e-commerce, a comprehensive normative system, can assist enterprise rules, establish a system and system related to national enterprises through multi-faceted convergence, and guide the smooth development of production and management.

\section{Conclusions}

This paper combines the future development trend of e-commerce, discusses the development space of electronic centralized procurement management from two aspects of advantages and disadvantages, and discusses new ideas for the transformation of electronic centralized procurement. Enterprises should integrate procurement operations as early as possible, optimize procurement procedures, and improve the efficiency and quality of centralized procurement. In theory, the development direction of e-commerce has yet to be demonstrated. Therefore, the research work in this paper aims to make a preliminary exploration for the development of electronic centralized procurement.

\section{References}

[1] Jinghua Xiao, Kang Xie, Yao Wu, Xuehua Liao, 2015, From partner to consumer oriented supply chain transformation, Management World, (4), pp.137-154.

[2] LushengLin, 2009, Analysis of the advantages of e-procurement and the implementation of e-procurement strategy, Logistics and purchasing research, (2), pp.134-135.

[3] Qiaoyan Cao, 2007, Research on the application mode of e-procurement for small and medium sized enterprises, CO-Operative Economy\&Science, (5), pp.95-96

[4] WeiXiong, Ming Xu, 2006, Procurement and warehousing management, Higher Education Press, Beijing,

[5] Yao Li, Mingyan Li, 2006, The development and case study of e-procurement in Chinese Enterprises, Proceedings of the Symposium on modern industrial engineering and management (MIEM'06), Science Press, Beijing.

[6] Yue Zhang, Weidong Chen, Zhiping Fan, 2004, Electronic centralized purchasing mode and example analysis, Modern Management Science, (9), pp.6-10. 